\title{
Optimality in self-organized molecular sorting
}

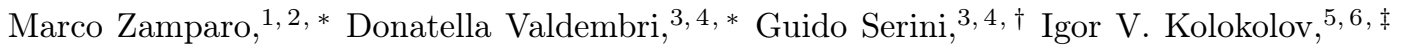 \\ Vladimir V. Lebedev, ${ }^{5,6,8}$ Luca Dall'Asta, 1,7,8,2, and Andrea Gamba ${ }^{1,2,8, * * *}$ \\ ${ }^{1}$ Institute of Condensed Matter Physics and Complex Systems, \\ Department of Applied Science and Technology, Politecnico di Torino, \\ Corso Duca degli Abruzzi 24, 10129 Torino, Italy \\ ${ }^{2}$ Italian Institute for Genomic Medicine c/o Candiolo Cancer Institute, \\ Fondazione del Piemonte per l'Oncologia (FPO), \\ Istituto di Ricovero e Cura a Carattere Scientifico (IRCCS), Candiolo, 10060 Torino, Italy \\ ${ }^{3}$ Department of Oncology, University of Torino School of Medicine, Candiolo, 10060 Torino, Italy \\ ${ }^{4}$ Candiolo Cancer Institute, Fondazione del Piemonte per l'Oncologia (FPO), \\ Istituto di Ricovero e Cura a Carattere Scientifico (IRCCS), Candiolo, 10060 Torino, Italy \\ ${ }^{5}$ L.D. Landau Institute for Theoretical Physics, 142432, \\ Moscow Region, Chernogolovka, Ak. Semenova, 1-A, Russia \\ ${ }^{6}$ National Research University Higher School of Economics, 101000, Myasnitskaya 20, Moscow, Russia \\ ${ }^{7}$ Collegio Carlo Alberto, Via Real Collegio 30, 10024 Moncalieri, Italy \\ ${ }^{8}$ Istituto Nazionale di Fisica Nucleare (INFN), Italy
}

\begin{abstract}
We introduce a simple physical picture to explain the process of molecular sorting, whereby specific proteins are concentrated and distilled into submicrometric lipid vesicles in eukaryotic cells. To this purpose, we formulate a model based on the coupling of spontaneous molecular aggregation with vesicle nucleation. Its implications are studied by means of a phenomenological theory describing the diffusion of molecules towards multiple sorting centers that grow due to molecule absorption and are extracted when they reach a sufficiently large size. The predictions of the theory are compared with numerical simulations of a lattice-gas realization of the model and with experimental observations. The efficiency of the distillation process is found to be optimal for intermediate aggregation rates, where the density of sorted molecules is minimal and the process obeys simple scaling laws. Quantitative measures of endocytic sorting performed in primary endothelial cells are compatible with the hypothesis that these optimal conditions are realized in living cells.
\end{abstract}

Keywords: protein sorting, phase separation, self-organization, physical kinetics, scaling laws, lattice gas

Introduction To counter the homogenizing effect of diffusion, eukaryotic cells developed an elaborate system to sort and distill specific proteins into submicrometric lipid vesicles, that are then transported towards appropriate intracellular destinations by active mechanisms involving molecular motors [1, 2]. Molecule sorting takes place on the plasma membrane, on inner membrane bodies (endosomes) and in the Golgi membrane network. Common biochemical principles involving the action of specialized proteins that promote membrane bending and fission [3 7] underlie molecular sorting in these different locations [1, 2, 8]. But can molecular sorting be understood as a sistemic process, beyond the molecular detail? Self-aggregation processes driven by reinforcing feedback loops lead to the formation of submicrometric domains enriched in specific lipids and proteins, and are ubiquitous on cell membranes [see Refs. 9 12, and references therein]. Moreover, the formation of such molecular aggregates, which can be likened to a phase separation process, that, along with sorted cargo, may involve several adaptor, membrane-bending and fission-inducing proteins, has been observed to precede and induce vesicle nucleation [13, 14, and evidences suggest that protein crowding by itself can drive membrane bending and vesicle nucleation [15 20] by making these processes energetically favorable [21-26]. Altogether, these observations suggest that sorting may be a universal process emerging from the coupling of two main components: a) the self-aggregation of localized protein microdomains, and b) vesicle nucleation. In this scheme, molecules that diffuse on a membrane can aggregate into localized enriched domains that grow due to molecule absorption. When a domain reaches a sufficiently large size, its biochemical constituents locally induce higher membrane curvature and the consequent nucleation and detachment of a small vesicle. The newly generated vesicle is constitutively enriched in the biochemical factors of the engulfed domain, resulting in a spontaneous distillation process. Here, we formulate a phenomenological theory of molecular sorting based on these principles and compare its predictions with numerical simulations of a lattice-gas model and quantitative measures of the kinetics of endocytic sorting in living cells. Our analysis suggests that higher sorting efficiency is realized when the number of sorting domains is minimized, a situation taking place for intermediate levels of the self-aggregation strength. Our quantitative measures suggest that such optimal conditions may be realized in living cells.

Phenomenological Theory We describe a situation where molecules arrive on a membrane region, diffuse and aggregate into localized enriched domains, and these domains are removed from the membrane, after reaching 
a characteristic size $R_{E}$, through the formation of small separate lipid vesicles. In this picture, sorting domains coexist in a statistically stationary state with a continuously repleted dilute solution, or "gas", of molecules that diffuse freely on a membrane. This is reminiscent of two-dimensional diffusion-limited aggregation (DLA) [27] or the related Hele-Shaw problem 28. However, in the advanced stages of DLA large fractal clusters are formed [27], while in our problem the presence of the cutoff length $R_{E}$ restricts the domain size, and domain shapes remain approximately round. Here, as in the classical framework of Lifshitz-Slezov (LS) theory [29, 30], domains of size $R$ larger than a critical value $R_{\text {c grow ir- }}$ reversibly by means of the absorption of single molecules diffusing towards them. This mechanism is expected to provide the dominant contribution to the absorption dynamics for sufficiently small average molecule density $\bar{n}$ in the gas. For domains with sizes much larger than the critical size $R_{\mathrm{c}}$, the density $n_{0}$ near the domain boundary is independent of its size. When the typical inter-domain distance $L$ is much larger than $R_{E}$, the difference $\Delta n$ in molecule density between the regions farther away and closer to the domain boundaries is approximately given by $\bar{n}-n_{0}>0$. Contrary to LS theory, in which domains can grow arbitrarily in time and $\Delta n$ tends to zero as time grows, here the size of domains removed from the system introduces a cutoff length $R_{E} \gg R_{\mathrm{c}}$, and, in the statistically stationary regime, $\Delta n$ is kept finite by the continuous influx of particles into the system.

The quasi-static profile of the density of freely diffusing molecules in the vicinity of a circular domain of size $R$ is obtained solving the Laplace equation with Dirichlet boundary conditions:

$$
n(r)=n_{0}+\frac{\ln r / R}{\ln L / R} \Delta n,
$$

where $r$ is the distance from the domain center. Deviations of the domain shape from circularity produce rapidly decaying higher multipole contributions that may be neglected in the main approximation.

The domain grows due to the flux $\Phi_{R}$ of molecules from the gas, which can be found by integrating the flux density $-D \nabla n$ over a circle of radius $r \gg R$ :

$$
\Phi_{R}=\left.2 \pi R D \partial_{r} n(r)\right|_{r=R}=\frac{2 \pi D \Delta n}{\ln (L / R)},
$$

where $D$ is the diffusion coefficient of isolated molecules. From (2) one obtains the dynamic equation for domain growth, $\dot{R}=A_{0} D \Delta n /(R \ln (L / R))$, where $A_{0}$ is the area occupied by a molecule in the domain, and the domain size $R$ is such that the domain area is $\pi R^{2}$.

Abstracting from complicated molecular details, the mesoscopic effects of vesicle extraction will be encoded in a single parameter, the rate $\gamma(R)$ by which domains of size $R$ are removed from the system. If $N(t, R) \mathrm{d} R$ is the number of domains per unit area with size between $R$ and $R+\mathrm{d} R$, the number density $N(t, R)$ satisfies the Smoluchowski equation

$$
\frac{\partial N}{\partial t}+\frac{\partial}{\partial R}(\dot{R} N)=-\gamma(R) N
$$

A stationary solution of 3 is

$$
N_{\mathrm{st}}(R)=\frac{J R \ln (L / R)}{D \Delta n} \exp \left[-\int_{0}^{R} \mathrm{~d} r \frac{r \ln (L / r) \gamma(r)}{A_{0} D \Delta n}\right] .
$$

We assume that the extraction rate $\gamma(R)$ is negligible for $R<R_{E}$ and strongly suppresses $N_{\mathrm{st}}(R)$ for $R>R_{E}$, where $R_{E}$ is the characteristic size of the domains that are extracted from the membrane. The factor $J$ in Eq. (4) is determined by noticing that, in the stationary regime, the average flux $\int \Phi_{R} N_{\text {st }}(R) \mathrm{d} R$ must equate the incoming flux of molecules per unit area $\phi$ (one of the control parameters of the theory), thus giving $J \sim \phi / R_{E}^{2}$. In the region $R<R_{E}$ where $\gamma(R)$ is negligible, Eq. (4) shows that the distribution $N_{\text {st }}(R)$ has a universal behavior characterized by a linear growth with logarithmic corrections. The present phenomenological approach is applicable if the inequality $R_{E}^{2} \gg A_{0}$ is satisfied. This condition also justifies the quasi-static approach leading to Eq. 11.

The efficiency of the sorting process can be measured in terms of the average residence time $\bar{T}$ of a molecule on the membrane system. For absorbing domains, this is the sum of the average time $\bar{T}_{\mathrm{f}}$ required by the molecule to reach a domain by free diffusion and be absorbed, and the average time $\bar{T}_{\mathrm{d}}$ spent inside that domain until the extraction event. For evenly distributed domains, the first contribution $\bar{T}_{\mathrm{f}}$ is inversely proportional to the average number $N_{\mathrm{d}}$ of domains per unit area, where $N_{\mathrm{d}}=\int \mathrm{d} R N_{\text {st }}(R) \sim \phi /(D \Delta n)$, giving $\bar{T}_{\mathrm{f}} \sim 1 /\left(D N_{\mathrm{d}}\right) \sim \Delta n / \phi$. In its turn, the average time spent by a molecule in a domain can be estimated as $\bar{T}_{\mathrm{d}} \sim R_{E}^{2} /\left(A_{0} \Phi_{R}\right) \sim R_{E}^{2} /\left(D A_{0} \Delta n\right)$, where (2) was used.

The rate of formation of new domains can be estimated as $\mathrm{d} N_{\mathrm{d}} / \mathrm{d} t=C D \bar{n}^{2}$, where $C$ is a dimensionless quantity characterizing the efficiency of absorption of single molecules by the germ of a domain. In the stationary condition this rate is equal to $N_{\mathrm{d}} / \bar{T}_{\mathrm{d}}$, therefore

$$
\bar{n} \sim\left(\frac{\phi A_{0}}{C D R_{E}^{2}}\right)^{1 / 2}
$$

Assuming $n_{0} \lesssim \Delta n$ we get $\Delta n \sim \bar{n}$ and then

$$
\bar{T}_{\mathrm{d}} \sim C^{1 / 2} \frac{R_{E}^{3}}{(D \phi)^{1 / 2} A_{0}^{3 / 2}}, \quad \bar{T}_{\mathrm{f}} \sim C^{-1 / 2} \frac{A_{0}^{1 / 2}}{(D \phi)^{1 / 2} R_{E}}
$$

The sum $\bar{T}=\bar{T}_{\mathrm{d}}+\bar{T}_{f}$, as a function of $C$, has a minimum 
in $C \sim A_{0}^{2} / R_{E}^{4} \ll 1$, where

$$
\begin{aligned}
\bar{T}_{\mathrm{f}} \sim \bar{T}_{\mathrm{d}} \sim \frac{R_{E}}{\left(D A_{0}\right)^{1 / 2} \phi^{1 / 2}}, \\
\bar{n} \sim \Delta n \sim \frac{\phi^{1 / 2} R_{E}}{\left(D A_{0}\right)^{1 / 2}} .
\end{aligned}
$$

Therefore, the scaling relations $(7 / 8)$ identify the dynamical regime in which molecular sorting is most efficient. The density of molecules accumulated in the domains is

$$
\rho_{\mathrm{d}} \sim N_{\mathrm{d}} R_{E}^{2} / A_{0} \sim C^{1 / 2} \frac{\phi^{1 / 2} R_{E}^{3}}{D^{1 / 2} A_{0}^{3 / 2}} .
$$

Thus, also the total density $\rho=\bar{n}+\rho_{\mathrm{d}}$ has a minimum for $C \sim A_{0}^{2} / R_{E}^{4}$, and the minimal value of $\rho$ is again determined by the estimate (8).

Lattice-gas model and numerical results To further explore the role of molecule self-aggregation in the distillation process, and to probe the behavior of the sorting process over a wide range of parameter values, we introduce here a minimal lattice-gas model of molecular sorting, without any attempt at a complete description of the complex biochemical and physical details implied in the process of vesicle budding and removal.

We represent the lipid membrane as a two-dimensional square lattice with periodic boundary conditions, where each lattice site can host at most a single molecule [31. The system evolves according to a Markov process that comprises the following three elementary mechanisms: 1) Molecules from an infinite reservoir arrive and are inserted on empty sites with rate $k_{I}$. 2) Then, molecules can perform diffusive jumps to an empty neighboring site with rate $k_{D} / g^{\# n n}$, where $g>1$ is a dimensionless aggregation coefficient and \#nn is the number of molecules neighboring the site originally occupied by the jumping molecule. 3) Finally, molecules are extracted from the system by the simultaneous removal of all connected molecule clusters, if any, that contain a completely filled square of linear size $\ell$, with $\ell^{2} \sim R_{E}^{2} / A_{0}$ (for a formal mathematical definition see SM). The stationary properties of the model depend on only two parameters, the ratio $k_{I} / k_{D}$ and the aggregation coefficient $g$. In what follows, areas are measured in units of a lattice site, therefore $A_{0}=1$, and the particle densities $\rho, n$ are dimensionless quantities.

The statistically stationary state of the model was investigated numerically. Fig. 1(a) shows that the stationary density of molecules $\rho$ is low for intermediate values of $g$, where a dilute gas of free molecules coexists with growing domains. In this region, the fraction of free molecules decreases as $g$ is increased, and the total molecule density has a minimum. The neighborhood of this minimum corresponds to the region previously found from the analysis of the phenomenological theory, which is likely the most interesting from the biological point of view. In Fig. 1 (b) the total density $\rho$ is decomposed into the contributions of freely diffusing molecules and of the molecules which are part of sorting domains. When $g$ is increased, the density of freely diffusing molecules decreases, while the number of molecules in the domains increases, leading to the appearance of a minimum of $\rho$ (Fig. 1(b), white circles) at intermediate values of the aggregation coefficient $g$. In this region, we computed numerical scaling relations with respect to the incoming flux per unit site $\phi=k_{I}(1-\rho)$, finding good agreement with the theoretical predictions 779 ) (Fig. 1.(c)-(d) and figure legend).

To characterize the efficiency of the sorting process we computed numerically the sorting rate $\bar{T}^{-1}=\phi / \rho$ (see SM and Ref. 32]) in terms of the physically meaningful parameters $\phi$ and $g$ [33. Fig. 1 (e) shows that $\bar{T}^{-1}$ increases monotonically with $\phi$, and that it exhibits a maximum as a function of $g$ at fixed $\phi$ (the dashed line in Fig. 1(e) marks the position of these maxima). In the optimal sorting region located around the maxima of $\bar{T}^{-1}$ distillation of molecular factors is most efficient.

The numerical evidence of a region of optimal sorting is in agreement with the phenomenological theory. This can be seen by considering that, in the framework of the numerical scheme, the efficiency $C$ of absorption of single molecules increases monotonically with $g$. Then the existence of the maximum of the sorting rate $\bar{T}^{-1}$ and of the minimum of the density $\rho$ observed in the numerical modeling appears as a natural consequence of the phenomenological theory. The contrasting behavior of the density of particles in the gas and in the domains (Fig. 1(b)) agrees with Eqs. (59).

Along with the residence time $\bar{T}$, which is a property of the stationary state, we considered also the characteristic adaptation time $T_{\text {ad }}$ needed by the membrane system to approach the stationary state after the sudden onset of a nonzero external stimulus. Numerical simulations show that $T_{\text {ad }}$ is directly correlated to $\bar{T}$ (see SM). Therefore, parameter values that correspond to optimal sorting in the stationary state are also those that provide faster response to changing environmental signals.

Comparison with experimental data It is interesting to check how experimental data compare with our general physical theory. Low-density lipoproteins (LDL) bind to LDL receptors (LDLR) on the plasma membrane in a 1:1 ratio, diffuse laterally, aggregate and are internalized in endocytic vesicles (see Refs. 34, 1 and SM, which includes additional Refs. 35-51). They provide a convenient experimental system whose behavior can be compared with the theory. We performed experiments of endocytic sorting of LDL on primary human endothelial cells incubated with LDL particles for 1 hour prior to imaging to allow them to reach a stationary state (see [52] and SM for details). LDL particles tagged with Alexa Fluor 488 green fluorescent dye were employed (SM). The local density of fluorescently-tagged molecules was quantified using total internal reflection fluorescence (TIRF) microscopy 

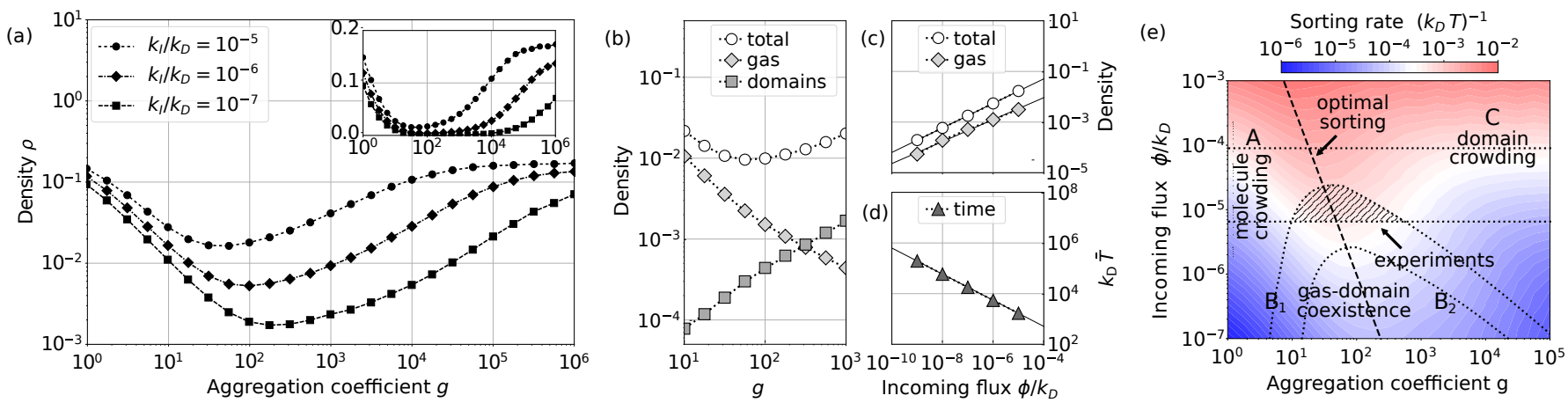

Figure 1. (a) Molecule density $\rho$ (time average in the statistically stationary state) as a function of the aggregation coefficient $g$, for $k_{I} / k_{D}=10^{-5}, 10^{-6}, 10^{-7}$. Inset: same quantities in log-linear scale. (b) Partial molecule densities as functions of the aggregation coefficient $g$. The density of the gas component decreases, while the number of molecules in the interior of sorting domains increases for growing $g$ at fixed $\phi / k_{D}=10^{-8}$; as a consequence, the total molecule density has a minimum at an intermediate value of $g$. (c,d) Numerical evidence of scaling relations. Straight lines are fitted with the laws $\rho \sim \phi^{a}, \bar{n} \sim \phi^{b}$, and $\bar{T} \sim \phi^{-c}$ with $a=0.48 \pm 0.01, b=0.46 \pm 0.02$, and $c=0.52 \pm 0.01$. (e) Nondimensionalized sorting rate $\left(k_{D} \bar{T}\right)^{-1}$ as a function of the aggregation coefficient $g$ and of the nondimensionalized incoming flux $\phi / k_{D}$. At fixed $\phi$, faster distillation takes place for intermediate values of $g$, thus showing the existence of a region of parameter space where sorting is optimal. In this qualitative phase diagram, $\mathrm{A}, \mathrm{C}$ are high-density phases, characterized by molecule crowding and domain crowding, respectively; $\mathrm{B}$ is a low-density phase where the average domain size is much less than the average interdomain separation and sorting domains coexist with freely-diffusing molecules. The dashed line marks maximal sorting at fixed flux $\phi$, and divides the $\mathrm{B}$ region into regions of less $\left(\mathrm{B}_{1}\right)$ and more dilute gas $\left(\mathrm{B}_{2}\right)$. Model parameters compatible with experimental values of molecule density and flux are shown as a shaded area.

(Fig. 2(a)), which allows to constrain the analysis to a thin layer of approximately $100 \mathrm{~nm}$ from the plasma membrane [53]. In these experimental conditions it was possible to observe a large number of endocytic events including the formation and detachment of LDL-enriched vesicles (Fig. 2(b,c)). Growing fluorescently-tagged domains were identified by automated image analysis. Assuming that LDL particles are distributed with approximately constant, uniform probability on the surface of sorting domains, and neglecting curvature corrections, the cumulative fluorescence intensity collected from a given domain is expected to be approximately proportional, in average, to the area of the domain. In order to fix the conversion factor, we assumed that the typical fluorescence intensity reached by growing domains just before their extraction corresponds to the size of mature endocytic vesicles, $R_{E} \sim 100 \mathrm{~nm}$ [18 (see SM). Histograms of domain sizes (Fig. 2(d)) show an approximately linear dependence of the frequency density on domain size for radii $R<R_{E}$, compatibly with the behavior predicted by Eq. (4). Assuming $\gamma(R)=0$ for $R<R_{E}$ and $\gamma(R)=\gamma_{0}$ for $R>R_{E}$, and fitting $\gamma_{0}$, Eq. (4) can be adapted to the experimental data also for $R>R_{E}$ (Fig. 2(d)).

We measured LDL surface density $\bar{\rho}=1.7 \cdot 10^{-2}$ $\left(\sigma_{\rho}=0.8 \cdot 10^{-2}\right)$ and flux $\bar{\phi}=2.8 \cdot 10^{-5}\left(\sigma_{\phi}=1.8 \cdot 10^{-5}\right)$ expressed, respectively, as fraction of the cell surface covered by sorting domains, and fraction of the cell surface extracted per second (see SM), compatibly with previous observations [34. The microscopic rate $k_{D}$ can be estimated as $D / A_{0}$, where $D=3 \cdot 10^{-3} \mu \mathrm{m}^{2} / \mathrm{s}$
$\left(\sigma_{D}=1.5 \cdot 10^{-3} \mu \mathrm{m}^{2} / \mathrm{s}\right)$ is the lateral diffusivity of LDL molecules [34, 54, and $A_{0} \sim 3 \cdot 10^{-3} \mu \mathrm{m}^{2}$ [55]. The relation $\bar{\phi}=k_{I}(1-\bar{\rho}) \simeq k_{I}$ allows then to estimate $k_{I} / k_{D} \sim 10^{-5}$. Fig. 1 (a) shows that for such parameter values, experimentally measured densities are attained in the physical model in the vicinity of the minima of the density $\rho$, i.e in the optimal region. On the phase diagram Fig. 1(e) the $g, \phi$ pairs compatible with the experiments are found at the intersection of the regions comprised between the numerically computed curves of equation $\rho=\bar{\rho} \pm \sigma_{\rho}$ and $\phi / k_{D}=\left(\bar{\phi} \pm \sigma_{\phi}\right) A_{0}\left(D \mp \sigma_{D}\right)^{-1}$ (dotted lines in Fig. 1(e)), and are situated in a neighborhood of the optimal region.

Discussion The observation of eukaryotic cells by fluorescence microscopy shows the hectic and apparently chaotic traffic of a myriad of submicrometric vesicles that transport lipids and proteins to disparate subcellular locations [56]. This restless movement takes place at significant energy cost, suggesting that it must be deeply relevant for cell life. Actually, to perform its vital tasks, such as feeding on nutrients, proliferating, migrating, and forming complex multicellular tissues, the cell has first of all to break its original symmetry [57]: in the process, each region of its outer and inner membranes becomes endowed with a specific chemical identity, that allows it to perform its peculiar functions $[58$. Vesicular traffic creates and sustains this broken-symmetry state: vesicles enriched in specific molecular factors are continuosly delivered to appropriate membrane regions to maintain their biochemical identity and to contrast 

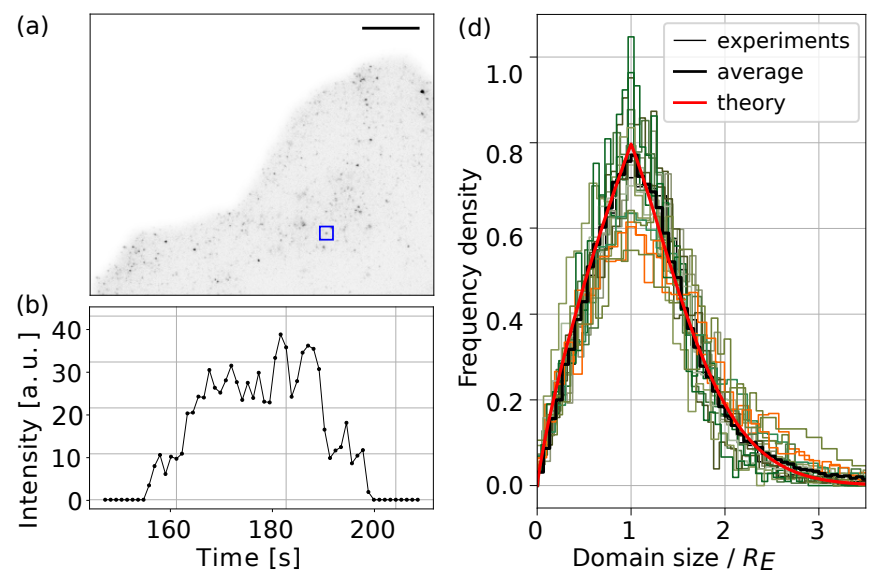

(c)

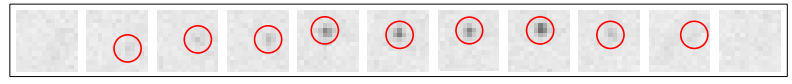

Figure 2. Sorting domains on the cell plasmamembrane. (a) TIRF micrograph of LDL sorting domains; scale bar: $10 \mu \mathrm{m}$; blue square: sample region of interest. (b) Time course of fluorescence intensity in the region of interest. (c) Snapshots of the region of interest separated by $\Delta t=5 \mathrm{~s}$ intervals, starting from $t=150 \mathrm{~s}$; red circles are centered around an automatically identified growing domain. (d) Frequency density of domain sizes: comparison of a fit of the theoretical curve (4) to data from 18 cells, pooled from two independent experiments. The domain size distribution computed from the complete experimental dataset is represented by the black line. Domain sizes were estimated as described in the text and SM.

the homogenizing effect of diffusion [59]. But what are the physical bases of this universal distillation mechanism? We have proposed here a scenario whereby molecular sorting emerges from the coupling of two spontaneous processes: a) phase separation induced by molecular self-aggregation [9 12, 30, 60], and b) vesicle nucleation. This view has solid bases in the physical chemistry of vesicular traffic, as several mechanisms have been identified that link the formation of molecular aggregates to the induction of membrane bending and vesicle nucleation [3 7, 16 18, 21,24]. From this general scenario, the following picture emerges: a continuously repleted gas of molecules diffusing towards multiple sorting centers, that grow due to molecular absorption and lead to the formation of vesicles in which a higher-than-average concentration of the given molecular factor has been distilled. The emergence of an optimal sorting regime at intermediate values of the aggregation strength then follows as a nontrivial effect of the physics of diffusion-limited aggregation on cell membranes, pointing out at the central role of self-aggregation in ordering the system. In our experiments coalescence of domains is rarely observed (see SM), suggesting that domains grow mainly by the absorption of laterally diffusing molecules from the surrounding molecule gas. The opposite regime, where do- mains mainly grow by coalescence, has been proposed to describe the formation of exit sites in the endoplasmic reticulum [61], suggesting that it would be interesting to develop a more general framework where both molecular aggregation and domain coalescence are relevant. Although here for simplicity we have considered the distillation of a single molecular factor, it is immediately evident that distinct clans of molecules endowed with high intra-clan affinity can separately aggregate in distinct enriched domains and be sorted in parallel. Clans of molecules that participate in common networks of reinforcing catalytic feedback loops are obvious candidates for the spontaneous formation of such enriched domains $[9-12,30,60$. By measuring the density of sorting domains and the sorting flux, we observed that LDL endocytosis in primary human endothelial cells kept in steady-state conditions takes place close to the optimal regime. It is then tempting to speculate that an evolutionary constraint may have led the proteins responsible for the distillation process to tune their activity around optimality, as maximal sorting efficiency may have provided selective advantage in terms of faster adaptation to rapidly varying environmental conditions.

MZ, LDA and AG thank Carlo Cosimo Campa, Jean Piero Margaria, Emiliano Descrovi and Emilio Hirsch for useful discussions. Numerical calculations have been made possible through a CINECA-INFN agreement providing access to resources on MARCONI at CINECA. The experimental research described in this work was supported by funding from Fondazione AIRC IG grants \#21315 (to GS) and \#20366 (to DV).

* Equal contribution.

† guido.serini@ircc.it

$\ddagger$ kolokol@itp.ac.ru

$\S$ lebede@itp.ac.ru

ฯ luca.dallasta@polito.it

** andrea.gamba@polito.it

[1] I. Mellman and W. J. Nelson, Nat. Rev. Mol. Cell Biol. 9, 833 (2008).

[2] S. Sigismund, S. Confalonieri, A. Ciliberto, S. Polo, G. Scita, and P. P. Di Fiore, Physiol Rev 92, 273 (2012)

[3] M. M. Kozlov, F. Campelo, N. Liska, L. V. Chernomordik, S. J. Marrink, and H. T. McMahon, Current Opinion in Cell Biology 29, 53 (2014)

[4] J. H. Hurley, E. Boura, L.-A. Carlson, and B. Różycki, Cell 143, 875 (2010)

[5] V. Haucke and M. M. Kozlov, J Cell Sci 131 (2018).

[6] M. Mettlen, P.-H. Chen, S. Srinivasan, G. Danuser, and S. L. Schmid, Annual review of biochemistry 87, 871 (2018)

[7] P. Bassereau, R. Jin, T. Baumgart, M. Deserno, R. Dimova, V. A. Frolov, P. V. Bashkirov, H. GrubmÃCEller, R. Jahn, H. J. Risselada, L. Johannes, M. M. Kozlov, R. Lipowsky, T. J. Pucadyil, W. F. Zeno, J. C. Stachowiak, D. Stamou, A. Breuer, L. Lauritsen, C. Simon, 
C. Sykes, G. A. Voth, and T. R. Weikl, J Phys D Appl Phys 51 (2018).

[8] L. M. Traub, Biochim Biophys Acta 1744, 415 (2005).

[9] M. Zamparo, F. Chianale, C. Tebaldi, M. CosentinoLagomarsino, M. Nicodemi, and A. Gamba, Soft Matter 11, $838(2015)$

[10] J. Halatek, F. Brauns, and E. Frey, Philosophical Transactions of the Royal Society B: Biological Sciences 373, 20170107 (2018)

[11] J. Berry, C. P. Brangwynne, and M. Haataja, Reports on Progress in Physics 81, 046601 (2018).

[12] A. S. Lyon, W. B. Peeples, and M. K. Rosen, Nat Rev Mol Cell Biol (2020)

[13] A. P. Liu, F. Aguet, G. Danuser, and S. L. Schmid, J Cell Biol 191, 1381 (2010).

[14] C. Puri, D. Tosoni, R. Comai, A. Rabellino, D. Segat, F. Caneva, P. Luzzi, P. P. Di Fiore, and C. Tacchetti, Molecular Biology of the Cell 16, 2704 (2005).

[15] P. Sens, L. Johannes, and P. Bassereau, Curr. Opin. Cell Biol. 20, 476 (2008).

[16 D. J. Busch, J. R. Houser, C. C. Hayden, M. B. Sherman, E. M. Lafer, and J. C. Stachowiak, Nat Commun 6, 7875 (2015)

[17] J. C. Stachowiak, E. M. Schmid, C. J. Ryan, H. S. Ann, D. Y. Sasaki, M. B. Sherman, P. L. Geissler, D. A. Fletcher, and C. C. Hayden, Nat. Cell Biol. 14, 944 (2012)

[18] M. Kaksonen and A. Roux, Nat. Rev. Molec. Cell Biol. 19, 313 (2018)

[19] N. S. Gov, Philosophical Transactions of the Royal Society B: Biological Sciences 373, 20170115 (2018)

[20] Z. Chen, E. Atefi, and T. Baumgart, Biophys J 111, $1823(2016)$

[21] S. Leibler, J. de Physique 47, 507 (1986)

[22] S. Leibler and D. Andelman, J. de Physique 48, 2013 (1987)

[23] A. F. Bitbol, L. Peliti, and J. B. Fournier, Europ. Phys. J. E 34 (2011)

[24] A. Banerjee, A. Berezhkovskii, and R. Nossal, Biophys. J. 102, 2725 (2012)

[25] S. A. Rautu, G. Rowlands, and M. S. Turner, Physical Review Letters 114, 098101 (2015).

[26] L. Foret and P. Sens, Proc. Natl. Acad. Sci. USA 105, 14763 (2008)

[27] A.-L. Barabasi and H. E. Stanley, Fractal Concepts in Surface Growth (Cambridge University Press, Cambridge, 1995).

[28] H. S. Hele-Shaw, Nature 58, 34 (1898)

[29] V. V. Slezov, Kinetics of First Order Phase Transitions (Wiley-VCH Verlag GmbH \& Co. KGaA, Weinheim, 2009).

[30] A. Gamba, I. Kolokolov, V. Lebedev, and G. Ortenzi, Phys Rev Lett 99, 158101 (2007).

[31] L. Bertini, A. D. Sole, D. Gabrielli, G. Jona-Lasinio, and C. Landim, J. Stat. Mech.: Theory and Experiment 2007, P07014 (2007)

[32] M. Zamparo, L. Dall'Asta, and A. Gamba, J. Stat. Phys. 174, $120(2019)$

[33] Since $\phi$ is a monotonically increasing function of the insertion rate $k_{I}$ (see SM), by a change of variable $\phi$ (which is directly observable) can be used as a control parameter in the place of $k_{I}$ (which is not observable).

[34] B. Goldstein, C. Wofsy, and G. Bell, Proc. Natl. Acad. Sci. USA 78, 5695 (1981).
[35] D. T. Gillespie, J. Comput. Phys. 22, 403 (1976).

[36] M. S. Brown, J. L. Goldstein, et al., Science 232, 34 (1986)

[37] T. Hevonoja, M. O. Pentikäinen, M. T. Hyvönen, P. T. Kovanen, and M. Ala-Korpela, Biochimica et Biophysica Acta (BBA) - Molecular and Cell Biology of Lipids 1488, 189 (2000)

[38] W. J. Schneider, in Biochemistry of Lipids, Lipoproteins and Membra edited by N. D. Ridgway and R. S. McLeod (Elsevier, 2016) 6th ed.

[39] T. L. Innerarity, R. E. Pitas, and R. W. Mahley, in Plasma Lipoproteins Part B: Characterization, Cell Biology, and Me Methods in Enzymology, Vol. 129 (Academic Press, 1986).

[40] M. Ehrlich, W. Boll, A. Van Oijen, R. Hariharan, K. Chandran, M. L. Nibert, and T. Kirchhausen, Cell 118, $591(2004)$

[41] M. Oheim, A. Salomon, A. Weissman, M. Brunstein, and U. Becherer, Biophysical Journal 117, 795 (2019).

[42] J. C. Waters and T. W. (Eds.), Quantitative Imaging in Cell Biology, 1st ed., Methods in Cell Biology Volume 123 (Academic Press, 2014).

[43] I. Khaw, B. Croop, J. Tang, A. Möhl, U. Fuchs, and K. Y. Han, Optics Express 26, 15276 (2018).

[44] M. A. Model, Current Protocols in Cytometry 37, 10.14.1 (2006)

[45] T. Lindeberg, Int. J. of computer vision 30, 79 (1998).

[46] F. Dekking, C. Kraaikamp, H. Lopuhaä, and L. Meester, A Modern Introduction to Probability and Statistics: Understanding (Springer, 2007).

[47] G. T. Dempsey, J. C. Vaughan, K. H. Chen, M. Bates, and X. Zhuang, Nature methods 8, 1027 (2011).

[48] O. Avinoam, M. Schorb, C. J. Beese, J. A. G. Briggs, and M. Kaksonen, Science 348, 1369 (2015)

[49] M. Kolb, R. Botet, and R. Jullien, Phys. Rev. Lett. 51, $1123(1983)$.

[50] T. A. Witten and L. M. Sander, Phys. Rev. Lett. 47, 1400 (1981).

[51] P. Meakin, Phys. Rev. Lett. 51, 1119 (1983).

[52] G. Mana, F. Clapero, E. Panieri, V. Panero, R. T. Böttcher, H.-Y. Tseng, F. Saltarin, E. Astanina, K. I. Wolanska, M. R. Morgan, M. Humphries, M. Santoro, G. Serini, and D. Valdembri, Nat. Commun. 7, 13546 (2016)

53] A. Picco and M. Kaksonen, Curr. Opin. Cell Biol. 53, 105 (2018)

[54] L. S. Barak and W. W. Webb, J. Cell Biol. 95, 846 (1982)

[55] Single molecule measurements show that endocytic vesicles host $\pi R_{E}^{2} / A_{0} \sim 10$ LDL molecules 62].

[56] B. Sönnichsen, S. De Renzis, E. Nielsen, J. Rietdorf, and M. Zerial, J. Cell Biol. 149, 901 (2000).

[57] R. Wedlich-Söldner and R. Li, Nat. Cell Biol. , 267 (2003)

[58] E. Rodriguez-Boulan and I. G. Macara, Nat. Rev. Molec. Cell Biol. 15, 225 (2014).

[59] A. Shewan, D. J. Eastburn, and K. Mostov, Cold Spring Harb. Perspect. Biol. , a004796 (2011).

[60] A. Gamba, A. de Candia, S. Di Talia, A. Coniglio, F. Bussolino, and G. Serini, Proc. Natl. Acad. Sci. USA 102, 16927 (2005)

61] S. Heinzer, S. Worz, C. Kalla, K. Rohr, and M. Weiss, J. Cell Sci. 121, 55 (2008).

[62] R. G. Anderson, M. S. Brown, and J. L. Goldstein, Cell 10, 351 (1977). 